\title{
Development of pDNA and Recombinant VSV Vectored Vaccines for Immunization Against HIV Steve Udem*‡
}

Address: Exploratory Viral Vaccine Research, Wyeth Vaccines, USA

Email: Steve Udem* - udems@wyeth.com

* Corresponding author $¥$ Presenting author

from 2005 International Meeting of The Institute of Human Virology Baltimore, USA, 29 August - 2 September 2005

Published: 8 December 2005

Retrovirology 2005, 2(Suppl I):S59 doi:I0.II86/I742-4690-2-SI-S59

Vaccines based on plasmid DNA (pDNA) or recombinant vesicular stomatitis virus (rVSV) vectors elicit HIV-1-specific cellular and humoral immune responses in small animal models. However, hurdles remain with each of these vaccine modalities before they can be considered for widespread clinical use. For pDNA vaccines, early clinical studies indicate that additional measures are needed to improve immunogenicity. For rVSV-based vaccines, issues related to the potential neurovirulence of the prototype vector represent a safety concern.

Adjuvant development and pDNA vector optimization are two important elements of current DNA vaccine research. Cytokine expression plasmids may function as potent adjuvants, and in fact, we have observed that plasmid-encoded IL-12 can substantially enhance immune responses in non-human primates. In addition, it is hypothesized that broad immune responses against multiple HIV antigens will be required to protect against infection and/or disease progression. Results from studies will be presented in which several pDNA vaccine designs were tested for their ability to elicit broadly reactive immune responses to multiple HIV antigens.

To address safety concerns related to use of rVSV vectors in humans, a range of further attenuated vector candidates has been developed and screened for neurovirulence in small animal models and nonhuman primates. Attenuated vectors have been identified that cause minimal CNS lesions after intracranial inoculation similar to those seen in control animals. Importantly, a number of these further attenuated vectors retained levels of immunogenicity in mice equivalent to prototype rVSV vectors, and have been advanced into nonhuman primate immunogenicity studies. 